\title{
Depolymerization of different organosolv lignins in supercritical methanol, ethanol and acetone to produce phenolic monomers
}

Xabier Erdocia ${ }^{1}$, Raquel Prado ${ }^{2}$, Javier Fernández-Rodríguez ${ }^{1}$, Jalel Labidi ${ }^{1 *}$

1. Chemical and Environmental Engineering Department. University of the Basque Country.

Plaza Europa 1, 20018 San Sebastian (Spain)

2. Department of Chemistry. Imperial College London. London SW7 2AZ, (United Kingdom)

*Corresponding author: E-mail: jalel.labidi@ehu.es ; Tel: +34 943017125

- Number of pages: 5

- Number of figures : 2

- Number of tables : 2 
Table S1. Compounds with more than $1 \%$ of the total chromatogram area identified in the pyrolyisis of FL and chars recovered after depolymerisation reactions with the three different solvents.

\begin{tabular}{|c|c|c|c|c|c|}
\hline \multirow[b]{2}{*}{ Compound } & \multirow[b]{2}{*}{$\begin{array}{c}\mathrm{RT} \\
(\min )\end{array}$} & \multicolumn{4}{|c|}{$\%$ of chromatogram area } \\
\hline & & $\mathrm{FL}$ & $\begin{array}{l}\text { Char FL } \\
\text { acetone }\end{array}$ & $\begin{array}{l}\text { Char FL } \\
\text { ethanol }\end{array}$ & $\begin{array}{c}\text { Char FL } \\
\text { methanol }\end{array}$ \\
\hline Toluene & 4.057 & --- & 1.69 & 2.17 & --- \\
\hline Furfural & 4.987 & 1.10 & --- & --- & --- \\
\hline Phenol & 7.420 & --- & 1.71 & 1.53 & 1.09 \\
\hline Limonene & 8.285 & --- & 1.80 & 1.51 & --- \\
\hline 2-methylphenol & 8.648 & 2.52 & 1.19 & 1.33 & --- \\
\hline 4-methylphenol & 8.972 & --- & 3.01 & 2.58 & --- \\
\hline 2-methoxyphenol & 9.284 & 1.56 & 4.19 & 4.18 & --- \\
\hline 2,4-dimethylphenol & 10.289 & --- & 1.32 & 1.38 & 1.35 \\
\hline 2-methoxy-4-methylphenol & 11.334 & --- & 5.35 & 6.18 & --- \\
\hline 3-methyl-1,2-benzenediol & 13.067 & --- & 1.86 & 2.53 & --- \\
\hline 3-methoxy-1,2-Benzenediol & 13.222 & --- & 3.58 & 4.85 & 3.26 \\
\hline 4-methyl-1,2-benzenediol & 14.043 & --- & --- & 2.02 & 1.03 \\
\hline Syringol & 15.995 & 4.26 & 4.11 & 4.27 & 3.64 \\
\hline 3,4-dimethoxyphenol & 16.225 & --- & 1.01 & 1.43 & 1.22 \\
\hline Vanillin & 17.161 & 1.16 & --- & --- & --- \\
\hline Vanillic acid & 18.143 & 1.12 & 2.14 & 2.64 & 2.90 \\
\hline Isoeugenol acetate (E) & 18.220 & 1.38 & --- & --- & --- \\
\hline Acetovanillone & 18.917 & 1.14 & --- & --- & --- \\
\hline Guaiacylacetone & 19.69 & 1.24 & --- & --- & --- \\
\hline Lauric acid & 20.084 & 2.51 & 1.53 & --- & --- \\
\hline Acetoveratrone & 20.210 & 1.94 & --- & --- & --- \\
\hline $\begin{array}{l}\text { 2,4'-Dihydroxy-3'- } \\
\text { methoxyacetophenone }\end{array}$ & 20.580 & 1.98 & --- & --- & --- \\
\hline Syringaldehyde & 21.591 & 1.83 & --- & --- & --- \\
\hline Methoxyeugenol & 22.111 & 3.47 & --- & --- & --- \\
\hline Palmitic acid & 24.941 & 7.64 & 3.87 & 5.42 & 2.70 \\
\hline Stearic acid & 26.875 & 2.92 & 5.22 & 1.65 & 2.37 \\
\hline Stigmastan-3,5-diene & 37.119 & --- & 1.08 & 1.08 & 1.00 \\
\hline
\end{tabular}


Table S2. Compounds with more than $1 \%$ of the total chromatogram area identified in the pyrolyisis of AFL and chars recovered after depolymerisation reactions with the three different solvents.

\begin{tabular}{|c|c|c|c|c|c|}
\hline \multirow[b]{2}{*}{ Compound } & \multirow[b]{2}{*}{$\begin{array}{c}\mathrm{RT} \\
(\min )\end{array}$} & \multicolumn{4}{|c|}{$\%$ of chromatogram area } \\
\hline & & AFL & $\begin{array}{l}\text { Char AFL } \\
\text { acetone }\end{array}$ & $\begin{array}{l}\text { Char AFL } \\
\text { ethanol }\end{array}$ & $\begin{array}{l}\text { Char AFL } \\
\text { methanol }\end{array}$ \\
\hline Toluene & 4.048 & --- & 1.13 & 3.03 & 1.56 \\
\hline Furfural & 4.992 & 1.31 & --- & --- & --- \\
\hline Phenol & 7.418 & --- & 1.18 & 3.08 & 1.58 \\
\hline 2-methylphenol & 8.649 & 2.37 & 1.19 & 2.01 & 1.66 \\
\hline 4-methylphenol & 8.978 & --- & 1.94 & 5.22 & 2.02 \\
\hline 2-methoxyphenol & 9.284 & 1.86 & 2.92 & 3.27 & 2.01 \\
\hline 2,5-dimethylphenol & 10.295 & --- & --- & 1.99 & --- \\
\hline 4-ethylphenol & 10.624 & --- & --- & --- & 1.16 \\
\hline 3,4-dimethylphenol & 10.687 & --- & --- & 1.58 & \\
\hline 2-methoxy-4-methylyphenol & 11.334 & --- & 4.51 & 5.54 & 2.22 \\
\hline 3-methyl-1,2-benzenediol & 13.069 & --- & 1.03 & 1.98 & 1.28 \\
\hline 3-methoxy-1,2-benzenediol & 13.228 & --- & 3.70 & 3.90 & 1.67 \\
\hline 4-methyl-1,2-benzenediol & 14.031 & ---- & --- & 1.72 & 1.88 \\
\hline 2-methoxy-4-vinylphenol & 14.967 & 1.79 & --- & --- & --- \\
\hline Syringol & 16.001 & 2.95 & 3.98 & 3.43 & 2.83 \\
\hline 3,4-dimethoxyphenol & 16.234 & --- & 1.23 & 1.18 & 1.10 \\
\hline Vanillin & 17.167 & 2.56 & --- & --- & --- \\
\hline Vanillic acid & 18.154 & 1.19 & 2.66 & 2.28 & 1.10 \\
\hline Isoeugenol acetate (E) & 18.224 & 4.24 & --- & --- & 2.22 \\
\hline D-Allose & 18.79 & 1.14 & --- & --- & --- \\
\hline Guaiacylacetone & 19.685 & 1.26 & --- & --- & --- \\
\hline Lauric acid & 20.084 & --- & 1.10 & --- & --- \\
\hline Acetoveratrone & 20.205 & 3.15 & --- & --- & --- \\
\hline $\begin{array}{l}\text { 2,4'-Dihydroxy-3'- } \\
\text { methoxyacetophenone }\end{array}$ & 20.580 & 1.26 & --- & --- & --- \\
\hline Syringaldehyde & 21.603 & 6.20 & --- & --- & --- \\
\hline Methoxyeugenol & 22.122 & 8.25 & --- & --- & --- \\
\hline Homosyringic acid & 22.994 & 2.75 & --- & --- & --- \\
\hline Palmitic acid & 24.941 & 9.95 & 3.04 & 2.95 & 1.48 \\
\hline Oleic acid & 26.708 & 2.73 & --- & --- & --- \\
\hline Stearic acid & 26.873 & 9.17 & 1.89 & 3.24 & 1.75 \\
\hline Stigmastan-3,5-diene & 37.119 & --- & 1.03 & 1.18 & 2.66 \\
\hline
\end{tabular}


Figure S1. Pyrograms of FL and chars recovered after depolymerization reactions with the three different solvents.
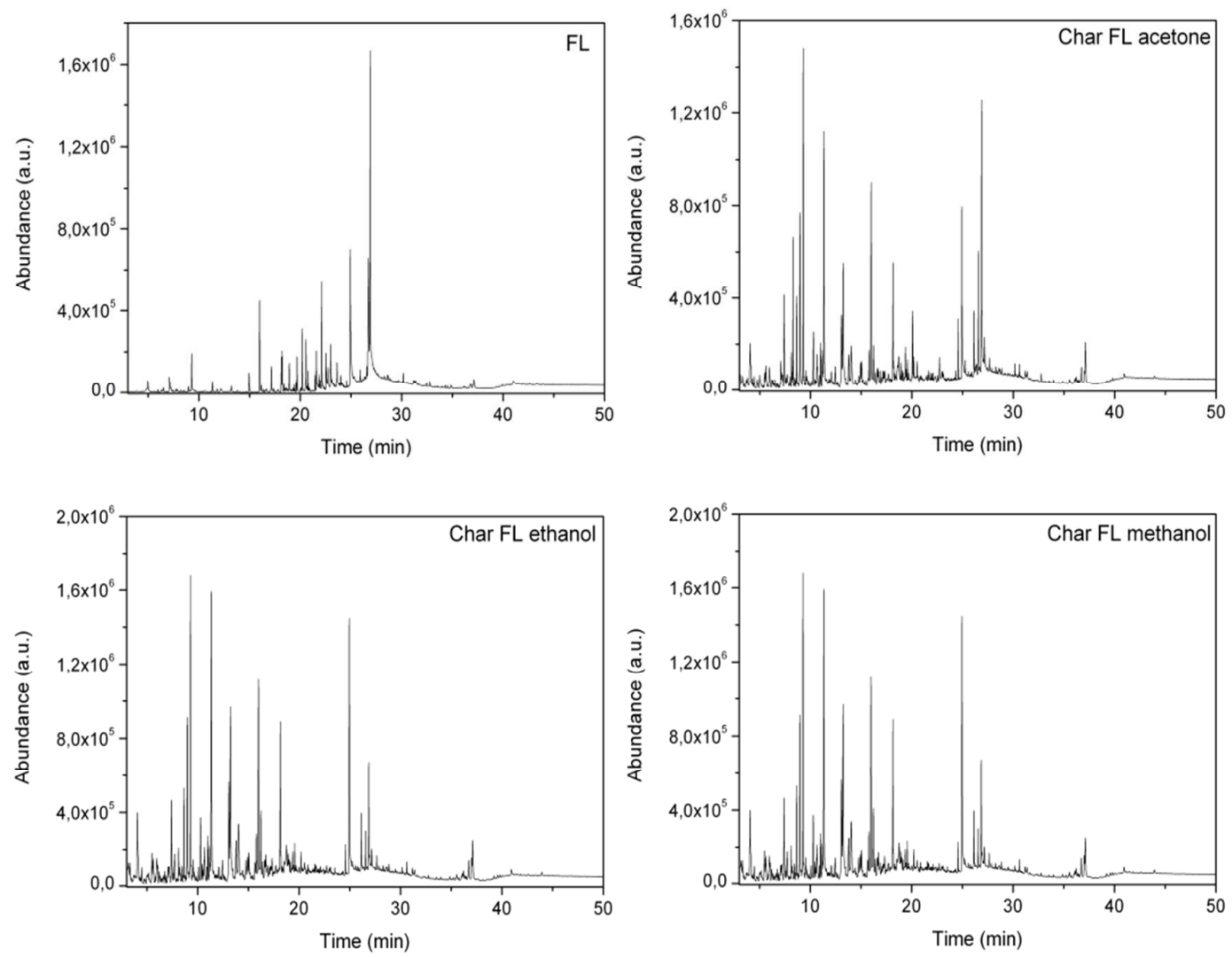
Figure S2. Pyrograms of AFL and chars recovered after depolymerization reactions with the three different solvents.
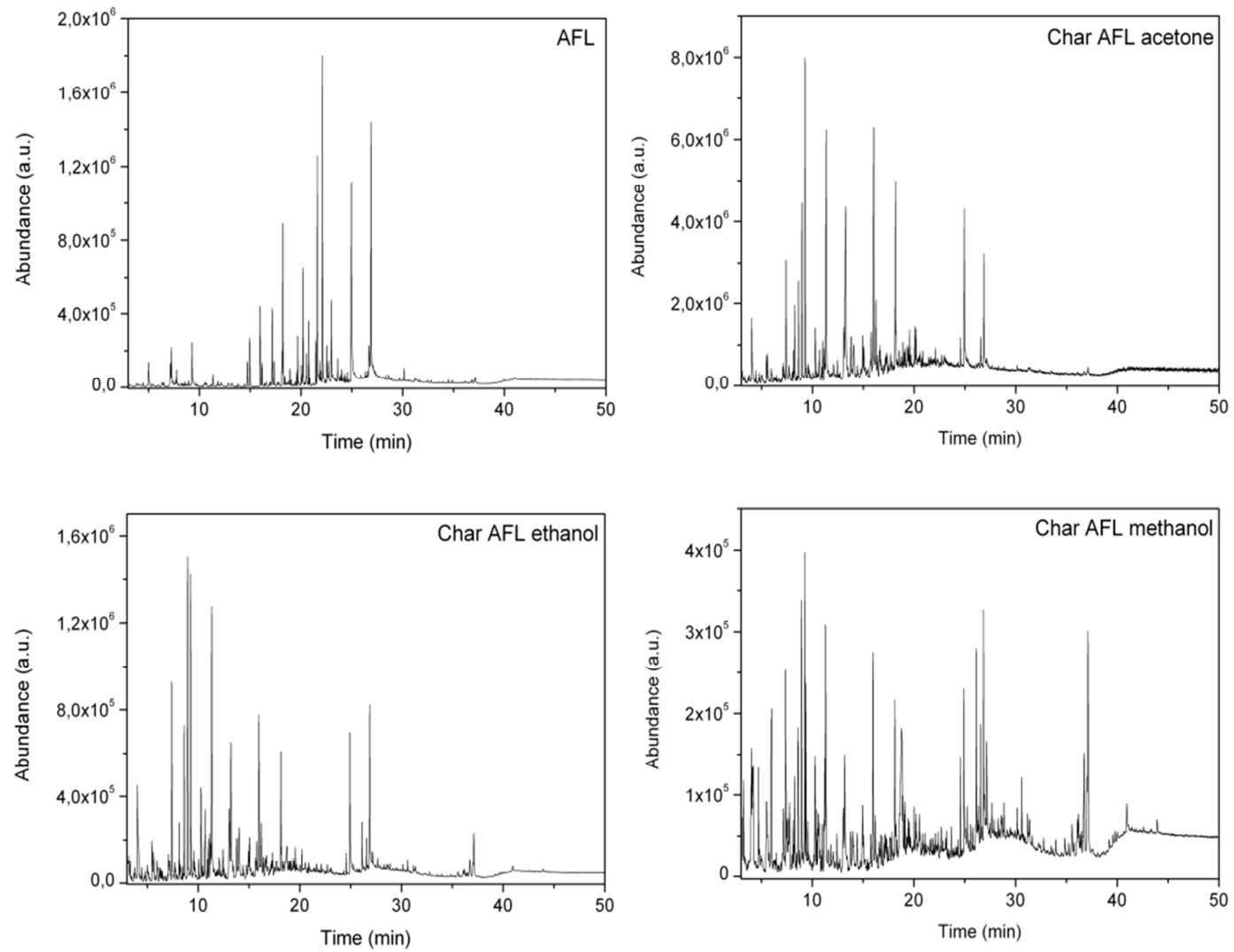